\title{
红磷/碳复合材料的制备及电化学性能研究
}

\author{
田丽媛, 姚志恒, 李 凤, 王永龙, 叶世海
}

(南开大学 新能源材料化学研究所, 天津 300071)

摘 要: 对比研究了不同制备方法、电解液组成和碳结构对红磷/碳复合材料电化学性能的影响。利用球磨法制备了 红磷/活性炭(AC)复合材料, 其较差的首次库伦效率和循环容量表明活性物质红磷没有得到有效利用。对多种电解 液进行了优选, 得到最优电解液为 $1 \mathrm{~mol} / \mathrm{L} \mathrm{LiPF}_{6}$ 的 EC/EMC/DMC $(1: 1: 1 \mathrm{~V} / \mathrm{V})$ 酯类电解液。通过气相沉积法制备了 红磷/导电碳黑(BP2000)和红磷/活性炭两种复合材料。利用热重分析(TGA)、X 射线衍射分析(XRD)、扫描电子显 微镜(SEM)、BET 比表面分析和循环伏安法(CV)对上述复合材料的形貌、结构和电化学性能进行了研究。结果表 明: 含磷量为 $45 \%$ 的红磷/活性炭复合材料充放电平台分别为 $1.0 \mathrm{~V}$ 和 $0.75 \mathrm{~V}$, 具有良好的可逆性。首次放电比容量和 充电比容量分别为 1500 和 $1200 \mathrm{mAh} / \mathrm{g}$, 库伦效率为 82.5\%。随后循环中库伦效率超过了 97.5\%。其 $1200 \mathrm{mAh} / \mathrm{g}$ 的循 环容量对应于 2.8 个电子的可逆反应。以第二次的稳定放电容量计算, 50 次循环后容量保持率为 $75.0 \%$ 。该复合材 料较高的循环容量和良好的循环稳定性受益于无定形的活性物质红磷在活性炭导电基底孔结构中, 特别是微孔中 的均匀分布。

关 键 词: 锂离子电池; 负极; 红磷; 气相沉积法; 多孔碳

中图分类号: TM912 文献标识码: A

\section{Synthesis and Electrochemical Performance of Red Phosphorus/Carbon Composites}

\author{
TIAN Li-Yuan, YAO Zhi-Heng, LI Feng, WANG Yong-Long, YE Shi-Hai \\ (Institute of New Energy Material Chemistry, Nankai University, Tianjin 300071, China)
}

\begin{abstract}
Effect of different fabrication methods, composition of electrolytes and various carbon substrates on the electrochemical performance of red phosphorus/carbon composites were studied. The red phosphorus (red P)/active carbon (AC) composites synthesized via ball milling method exhibited a low coulombic efficiency and cyclic capacity in the initial cycle, meaning poor utilization ratio of red P. It was found that the optimal electrolyte was $1 \mathrm{~mol} / \mathrm{L} \mathrm{LiPF}_{6}$ in an ethyl carbonate (EC)/ethyl methyl carbonate (EMC)/dimethyl carbonate (DMC) mixed solvent. Red P/conductive carbon black (BP2000) and red P/AC composites were synthesized by a vapor deposition method. The morphology, structure and electrochemical performance of the as-prepared composites were characterized by thermal gravity analysis (TGA), X-ray diffraction (XRD), scanning electron microscope (SEM), BET surface analysis and cyclic voltammetry (CV). Electrochemical results reveal that the red P/AC composite (45\%P) has a good reversibility with charge/discharge potential plateau at $1.0 \mathrm{~V}$ and $0.75 \mathrm{~V}$. The initial discharge/charge special capacity is 1500 and $1200 \mathrm{mAh} / \mathrm{g}$ (calculated based on the weight of composites), respectively, showing initial coulombic efficiency of 82.5\%. In the subsequent cycles the coulombic efficiency is over $97.5 \%$. The stable cyclic capacity is related to 2.8-electron reaction. Relying on the stable discharge
\end{abstract}

收稿日期：2014-11-24; 收到修改稿日期：2015-01-26

基金项目: 国家自然科学基金(51102137); 教育部创新团队(IRT13022)

National Natural Science Foundation of China(51102137); Innovative Research Team in University(IRT13022)

作者简介: 田丽媛(1989-), 女, 硕士研究生. E-mail: lytian@mail.nankai.edu.cn

通讯作者: 王永龙, 副教授. E-mail: ylwang@nankai.edu.cn 
capacity in the second cycle, the capacity retention is calculated to be $75.0 \%$ after 50 cycles. The as-prepared red P/AC composites exhibit high cyclic capacity and good cyclic stability, which can be attributed to the uniform dispersion of amorphous red $\mathrm{P}$ in the porous structure of AC substrate, especially in the micropores.

Key words: lithium ion batteries; anode; red phosphorous; vapor deposition method; porous carbon

为了满足人们在通信、便携式电子器械、电动汽 车 $(\mathrm{EV})$ 、混合式动力汽车 $(\mathrm{HEV})$ 等方面的要求, 高比能 量的锂离子电池(LIB)吸引了广泛关注 ${ }^{[1-4]}$ 。目前商用锂 离子正负极材料的实际比容量已接近其理论比容量, 因而探索具有更大理论比容量的新型电极材料是提高 下一代锂离子电池能量密度的关键之一 ${ }^{[5-7]}$ 。新型电极 材料中硫正极材料以较高的理论比容量 $(1672 \mathrm{mAh} / \mathrm{g})$ 而受到研究者的青睐 ${ }^{[8-9]}$ 。负极材料主要有锡基氧化 物 ${ }^{[10-11]}$ 、硅基化合物 ${ }^{[12]}$ 、过渡金属氧化物 ${ }^{[13]}$ 、金属 氮化物 ${ }^{[14]}$ 和金属磷化物 ${ }^{[15-17]}$ 等。其中硅负极材料因 拥有超高的理论比容量 $(4200 \mathrm{mAh} / \mathrm{g})$ 而得到广泛关 注 $^{[18-22]}$, 然而, 其较大的体积效应引起的容量衰减 以及较低的锂化电位造成的安全问题仍然面临着严 峻的考验 ${ }^{[18,23]}$ 。近年来, 红磷作为锂离子负极材料 逐步进入人们的视野。红磷作为电极材料具有以下 优势 ${ }^{[24-27]}$ : 红磷资源丰富可降低制造成本; 可实现 3 个电子的锂化反应, 理论比容量达 $2596 \mathrm{mAh} / \mathrm{g}$; 锂 化电位为 $0.75 \mathrm{~V}$, 该电位高于金属锂枝晶的沉积电位, 可以有效避免多次循环过程中由于锂枝晶的生成而 带来的安全问题。并且比 $\mathrm{Li}_{4} \mathrm{Ti}_{5} \mathrm{O}_{12}(1.5 \mathrm{~V})$ 和 $\mathrm{TiO}_{2}(2.0 \mathrm{~V})$ 的锂化电位低很多, 因而由红磷构成的锂离子电 池可以获得更高的工作电压, 进而获得更高的比 能量 ${ }^{[28-31]}$ 。由上可见,红磷作为锂离子负极材料具有 成本低、安全性好和比容量高等优势 $[25,32-35]$ 。然而, 红 磷是典型的非金属, 电子电导率很低, 约为 $10^{-14} \mathrm{~S} / \mathrm{cm}$ 。 由无定形红磷直接制备的电极的充放电容量和循 环性能较差。采用电导率较高的碳基底材料对导电 性差的电极材料进行改性是常用的改性方式 ${ }^{[36-37]}$ 。 文献[25, 38]报道了几种不同碳材料对磷电极性能的 影响, 说明碳基底对红磷的电化学性能有着极其重 要的影响。Qian 等 ${ }^{[25,35]}$ 利用高速振荡球磨法制备了 高性能的磷/碳复合材料, 显示出碳对红磷改性的有 效性。对于具有相似物化性质的硫正极材料, 电解液 的组成对其电化学性能也有较大影响 ${ }^{[39]}$ 。

本工作研究了高速行星球磨法制备的磷/碳复 合材料的电化学性能, 并对比了酯类和醚类两类电 解液中磷/碳复合材料的电化学行为。实验还以介孔 为主的导电碳黑和以微孔为主的活性炭为导电碳基 底, 利用不破坏碳基底孔结构的气相沉积法制备磷/ 碳复合电极材料, 并分析了形貌和结构, 研究了它
们在上述优选的电解液中的电化学性能。

\section{1 实验方法}

\section{1 磷/碳复合材料的制备和结构表征}

通过球磨法制备红磷/活性炭复合材料。将红磷 (0.45 g) 和活性炭 $(0.55 \mathrm{~g})$ 置于氧化锆球磨罐中, 使 用行星球磨机, 氩气保护下 $300 \mathrm{r} / \mathrm{min}$ 球磨 $24 \mathrm{~h}$ 得到 复合材料。通过气相沉积法制备了红磷/导电碳黑和 红磷/活性炭复合材料。先将红磷 (0.25 g) 和碳 $(0.25 \mathrm{~g})$ 置于 $50 \mathrm{~mL}$ 坩埚中, $\mathrm{Ar}$ 保护下升温到 $500^{\circ} \mathrm{C}$ 保持 $2 \mathrm{~h}$, 再降温到 $300^{\circ} \mathrm{C}$ 保持 $10 \mathrm{~h}$, 得到的材料用 无水乙醇和去离子水多次洗涤, 在 $60^{\circ} \mathrm{C}$ 真空干燥箱 中干燥 $24 \mathrm{~h}$ 。通过热重 (Mettler Toledo, TGA/DSC1) 测试, 证明两种复合材料中磷含量均为 $45 \%$ 。通过 XRD (Rigaku MiniFlex II)、SEM (Hitachi S-4800)、 BET (JW-BK instrument, Beijing, China)和 Raman (Renishaw, England) 分析材料的微观结构和形貌 特点。

\section{2 电池的组装和电化学性能的测试}

将磷/碳复合材料、乙炔黑 (AB) 和聚四氟乙烯 (PTFE)以重量比 7: 2: 1 混合, 用无水乙醇进行分散, 制浆和擀片, 再冲制成 $\phi 8 \mathrm{~mm}$ 的小圆片, $50^{\circ} \mathrm{C}$ 烘箱 中干燥 $24 \mathrm{~h}$, 烘干后的极片质量在 1.5 2.5 mg 之间。 在充满氩气的手套箱中制作测试电池，用金属锂作 为对电极和参比电极, 用微孔膜(Cellgard 2300)作为 隔膜, 使用铜簿作为半电池正负极的集流体。室温下 使用蓝电测试仪(Land CT2001C, China), 以电流密 度为 $100 \mathrm{~mA} / \mathrm{g}$ 的恒流充放电对电池进行测试, 充放 电的电位窗口是 $0.05 \sim 2.0 \mathrm{~V}\left(\mathrm{vs} . \mathrm{Li} / \mathrm{Li}^{+}\right)$。如未做特殊说 明，充放电比容量的计算均基于复合材料的总质量。 在电化学工作站(LK2005, China)上进行循环伏安曲线 的测试, 扫速为 $0.1 \mathrm{mV} / \mathrm{s}$ 。

\section{2 结果与讨论}

\section{1 球磨法制备的复合材料电化学性能}

图 1(a) 充放电曲线显示了该材料首次循环存 在较大的不可逆容量。材料首次放电 (锂化) 比容 量约为 $1400 \mathrm{mAh} / \mathrm{g}$ 。首次充电比容量迅速下降至 

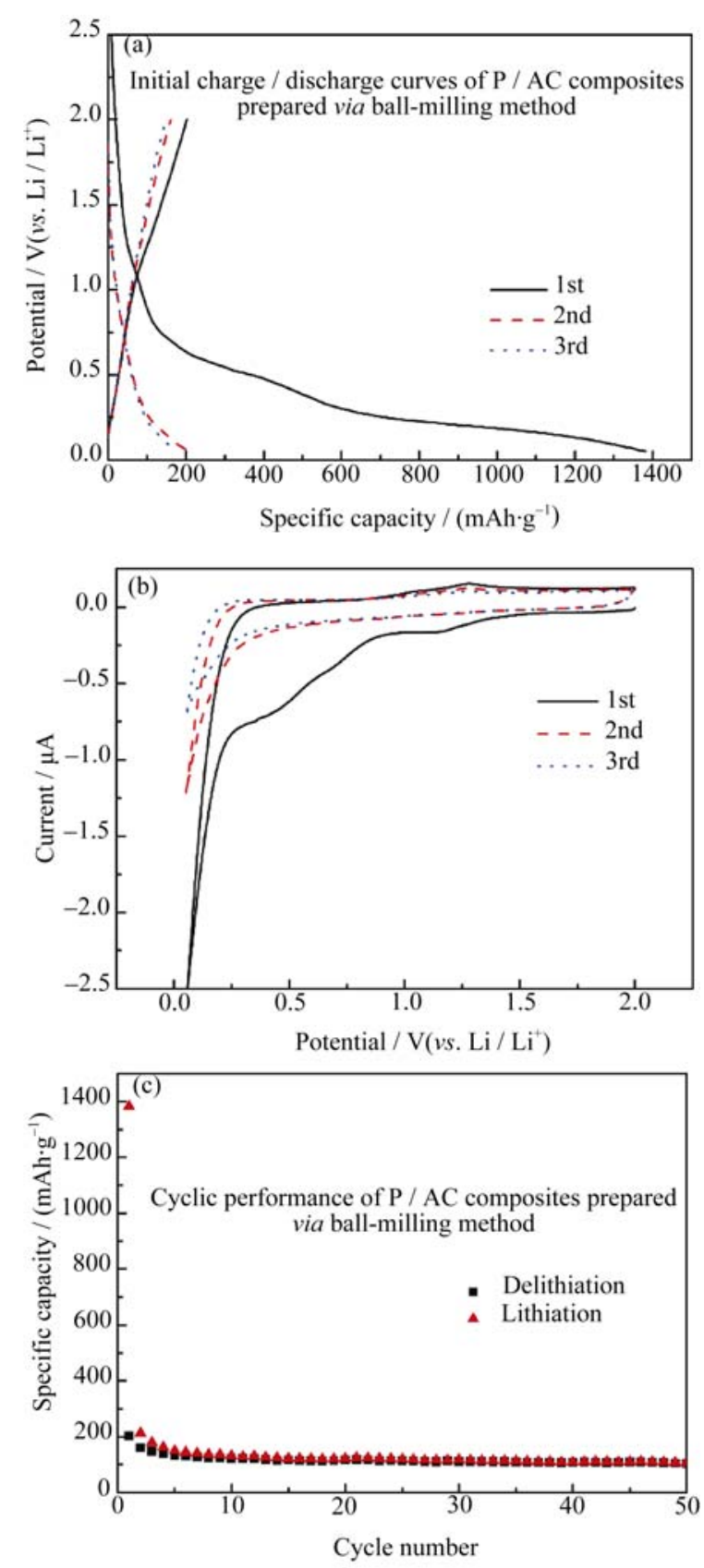

图 1 球磨法制备的含磷量 45\%的磷/活性炭复合材料的电化 学性能

Fig. 1 Electrochemical performance of $45 \%$ P/AC composites prepared by a ball-milling method

(a) CV curves, (b) Initial three charge (delithiation)-discharge (lithiation) curves of P/AC composite and (c) cycle performance of P/AC composite. Current density is $100 \mathrm{~mA} / \mathrm{g}$, the scan rate is $0.1 \mathrm{mV} / \mathrm{s}$

$200 \mathrm{mAh} / \mathrm{g}$ 。在随后的循环中, 比容量基本保持在 $200 \mathrm{mAh} / \mathrm{g}$ 。为了计算红磷的利用率, 活性炭、粘结 剂和乙炔黑(质量比为 $38.5: 10: 20$ )作为非活性组 分的电化学性能也做了测试(见附录图 S1)。经换算 发现在此复合材料中上述非活性材料首次放电比容 量贡献约为 $1174 \mathrm{mAh} / \mathrm{g}$, 其后循环比容量贡献约为 $200 \mathrm{mAh} / \mathrm{g}$ 。因而, 在首次放电过程中, 在复合材料 中红磷锂化贡献的容量仅为 $250 \mathrm{mAh} / \mathrm{g}$, 其活性物
质利用率约为 21\%。后续充放电循环过程中红磷基 本没有容量贡献。图 1(b)循环伏安曲线 $(\mathrm{CV})$ 首次还 原过程在 $1.0 \mathrm{~V}$ 以下有较大的还原电流, 随后的氧 化还原过程则几乎没有氧化还原峰。同样说明在首 周还原过程有红磷参与, 而随后的循环红磷没有氧 化还原反应，与充放电性能相对应。图 1(c)示出的 循环寿命有同样结果。因而经行星球磨制备的红磷/ 活性炭复合材料的反应活性较差, 红磷仅有少部分 在首次充放电过程中参与锂化反应，这部分锂化后 的红磷也不能可逆的再生成具有活性的红磷。图 2 显示了活性炭基底和红磷/活性炭复合材料的 SEM 形貌。活性炭在球磨前为微米级块状颗粒, 粒度分 布不均一, 颗粒边缘较锐利。图 2(b)显示了红磷/活 性炭复合材料的形貌,可以看出活性炭颗粒仍为粒 径不均一的块状, 颗粒尺寸变小, 边缘仍锐利, 除 此还存在团状微米尺度的红磷颗粒。因而, 可以认 为在球磨过程中碳颗粒尺寸变小, 与红磷只是简单 的机械混合, 形成微米级混合物。较大的红磷颗粒 没有得到有效利用。考虑到红磷本身较差的导电性 和无定形结构, 提高红磷的反应活性必须减小红磷 颗粒尺寸。振荡式高速球磨可以提供更大能量 (>1000 r/min), 制备尺寸更小的红磷颗粒, 从而提 高红磷的有效利用率 ${ }^{[25]}$ 。为了使红磷能够均匀分散, 本工作采用不破坏碳基底孔结构的气相沉积法, 将 红磷吸附在碳基底丰富的介孔和微孔中, 并利用孔 的限制控制红磷颗粒的大小, 以达到均匀分散和限 制磷颗粒粒径的目的。

\section{2 电解液对红磷/活性炭复合材料电化学性能 的影响}

鉴于硫磷同为导电性差的非金属单质，酯类和 醚类电解液对硫电极的影响可能同样适用于红磷电 极。图 3(a)和(d) 是使用电解液为 $1 \mathrm{~mol} / \mathrm{L} \mathrm{LiPF}_{6}$ 在 EC/DMC $(V / V$ 3: 7) 混合电解液中前三周充放电曲 线和循环性能曲线, 从图 3(a)可以看出, 首次放电 比容量为 $1400 \mathrm{mAh} / \mathrm{g}$, 随后充放电比容量下降到 $800 \mathrm{mAh} / \mathrm{g}$ 。从图 3(d)可以看到循环容量在 50 次循 环后逐步衰减到约 $600 \mathrm{mAh} / \mathrm{g}$ 。图 3(b)和(e)是使用

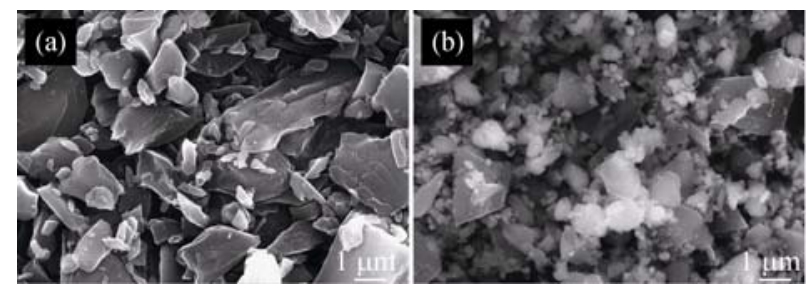

图 2 (a)活性炭和(b)球磨制备的红磷/活性炭复合材料扫描电 镜图片

Fig. 2 SEM images of AC (a) and red P/AC composites (b) 

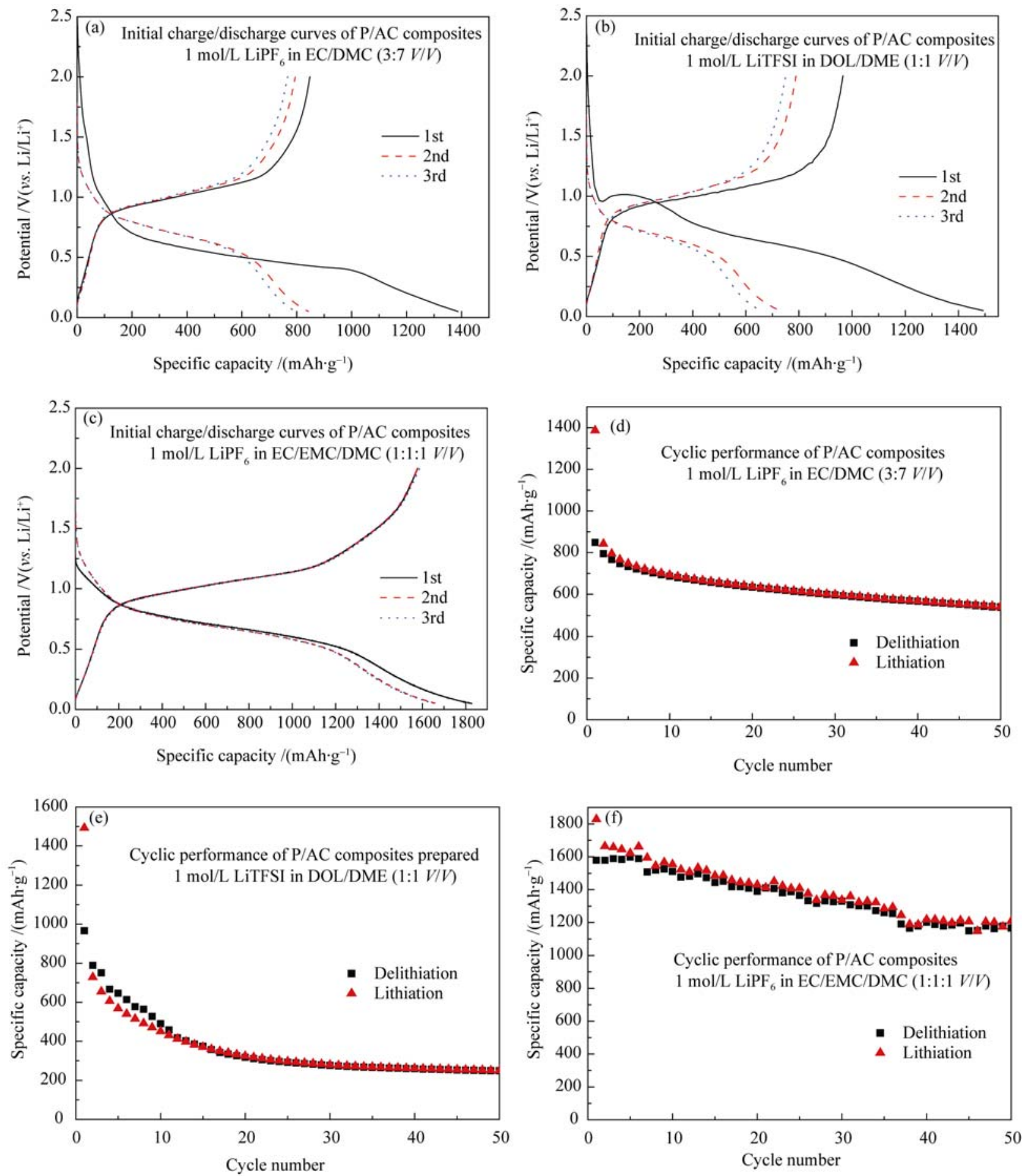

图 3 磷/活性炭复合材料在不同电解液中的初始三次充放电曲线 ( $\mathrm{a}, \mathrm{b}$ 和 $\mathrm{c}$ )及循环寿命曲线 (d, e 和 $\mathrm{f}$ )

Fig. 3 The initial three charge-discharge curves (a, b and c) and cycle performance (d, e and f) of P/AC composite in different electrolytes

电解液 $1 \mathrm{~mol} / \mathrm{L} \mathrm{LiTFSI}$ 在 DOL/DME (1: $1 \mathrm{~V} / \mathrm{V})$ 混合 溶液中的充放电曲线和循环性能曲线, 首次放电在 $1.0 \mathrm{~V}$ 出现了一个放电平台。在这个平台上电压出现 了短暂上升和下降, 这可能是由于材料极化产生 的。首周放电比容量为 $1450 \mathrm{mAh} / \mathrm{g}$, 首次充电比容 量为 $1000 \mathrm{mAh} / \mathrm{g}$ 。从图 3(e)的循环性能来看, 复合 材料在该电解液中前 20 次循环比容量快速衰减到
$300 \mathrm{mAh} / \mathrm{g}$, 随后循环性能较稳定, 50 次循环后比容 量为 $250 \mathrm{mAh} / \mathrm{g}$ 。图 3(c)和(f)是使用电解液为 $1 \mathrm{~mol} / \mathrm{L}$ $\mathrm{LiPF}_{6}$ 在 $\mathrm{EC} / \mathrm{EMC} / \mathrm{DMC}(1: 1: 1 \mathrm{~V} / \mathrm{V})$ 混合溶液中的充 放电曲线和循环性能曲线, 首次放电比容量约为 $1800 \mathrm{mAh} / \mathrm{g}$, 首次充电比容量是 $1650 \mathrm{mAh} / \mathrm{g}$, 首次 库伦效率是 $91.7 \%$ 。循环比容量衰减较缓慢, 到 50 次循环后比容量保持在 $1150 \mathrm{mAh} / \mathrm{g}$ 。第二次充放电 
之后的库伦效率在 $97.0 \%$ 以上。较高的库伦效率表 明在该电解液中, 磷/碳复合材料具有良好的可逆性, 有效利用率也较高, 但容量衰减仍较快。由上述分 析可知, $1 \mathrm{~mol} / \mathrm{L} \mathrm{LiPF}_{6}$ 的 EC/EMC/DMC (1: $\left.1: 1 \mathrm{~V} / \mathrm{V}\right)$ 混合酯类电解液是最适配的电解液。

\section{3 两种碳材料构筑的磷/碳复合材料的结构和 电化学性能}

通过气相沉积法制备了不同种碳 (导电碳黑 BP2000 和活性炭 AC) 的磷/碳复合材料。复合材料 中的磷含量通过热重分析得到, 两种复合物中的磷 含量均为 $45.0 \%$ (见附录图 S2)。图 4 显示了两种复 合材料红磷/导电碳黑和红磷/活性炭的 XRD 图谱, 可以看出磷/碳复合材料中的衍射峰与红磷的衍射 峰是相对应的, 在 $15.5^{\circ}$ 和 $34.1^{\circ}$ 分别出现了宽化的 衍射峰, 证明复合材料中的磷是以红磷形式存在的, 不是白磷或者黑磷。复合材料中也分别出现了无定 型碳的衍射峰, 也证明了碳的存在。

图 5 为导电碳黑、红磷/导电碳黑、活性炭和红 磷/活性炭的 SEM 照片。对比红磷气相沉积前后的 形貌, 可以看到颗粒粒径和尺寸分布均无较大变化, 可以认为红磷沉积并未生成独立的颗粒而造成负载 前后颗粒粒径和尺寸分布的变化。原活性炭颗粒边 缘较锐利, 经过红磷负载后, 复合材料颗粒边缘明 显针化, 这是由无定形红磷薄层负载在碳基底颗粒 上造成的。

表 1 是碳和复合材料的比表面积及孔结构参数, 通过 $\mathrm{N}_{2}$ 吸脱附曲线获得, 样品测试前在 $150^{\circ} \mathrm{C}$ 处理 $1 \mathrm{~h}$ 。BET 比表面积是在相对压力为 $0.05 \sim 0.5 \mathrm{P}$ 的范 围通过 BET 方程的线性部分计算得到, 微孔和介孔 的粒径分布由 Horvath-Kawazoe (HK) 和 BarrettJoyner-Halenda (BJH)方法计算。对于导电炭黑基底, BET 比表面积是 $1128.1 \mathrm{~m}^{2} / \mathrm{g}$, 负载磷后, 比表面积为 $275.2 \mathrm{~m}^{2} / \mathrm{g}$, 微孔体积从 $0.57 \mathrm{~cm}^{3} / \mathrm{g}$ 降到了 $0.08 \mathrm{~cm}^{3} / \mathrm{g}$,

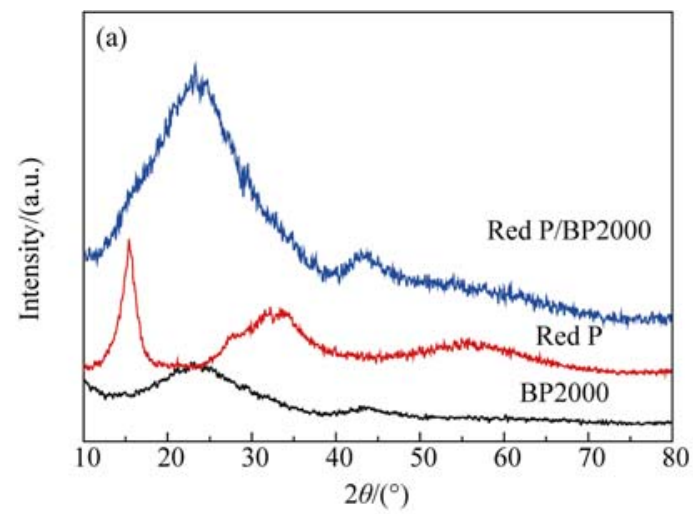

介孔体积从 $0.86 \mathrm{~cm}^{3} / \mathrm{g}$ 降到了 $0.50 \mathrm{~cm}^{3} / \mathrm{g}$ 。对于活性 炭基底, BET 比表面积是 $1528.2 \mathrm{~m}^{2} / \mathrm{g}$, 负载磷后, 比 表面积为 $127.1 \mathrm{~m}^{2} / \mathrm{g}$, 微孔体积从 $0.88 \mathrm{~cm}^{3} / \mathrm{g}$ 降到了 $0.02 \mathrm{~cm}^{3} / \mathrm{g}$, 介孔体积从 $0.46 \mathrm{~cm}^{3} / \mathrm{g}$ 降到了 $0.20 \mathrm{~cm}^{3} / \mathrm{g}$ 。 两种碳基底的微孔孔容都接近于 0 , 而介孔还有较 大剩余空间, 因此红磷优先在微孔中负载, 其次在 介孔中负载。由比表面积下降程度计算, 红磷/导电 碳黑复合材料中约有 $65 \%$ 的红磷负载在介孔内,而 对于红磷/活性炭复合材料则为 34\%。这是由于导电 碳黑和活性炭的介孔和微孔的占比不同, 导致红磷 在导电碳黑中以介孔为主, 而在活性炭里以微孔为 主。拉曼光谱表明红磷负载前后碳的 $G$ 峰和 $D$ 峰均 未发生位移(见附录图 S3), 说明两种复合材料中碳 和红磷没有较强的相互作用。

图 6(a)和(b)分别是红磷/导电碳黑和红磷/活性 炭复合材料的循环伏安曲线, 从图中可以看出两种 复合材料在三次扫描过程中都出现了一对氧化还原 峰。首次循环峰电位分别位于 0.55 和 $1.1 \mathrm{~V}$ 。第二 次循环后, 还原峰电位正移到 $0.6 \mathrm{~V}$, 且氧化和还原 峰的面积相近, 意味着两种复合材料都具有良好的 可逆性。结合空白碳首周较大的不可逆容量(见附录 图 S1)。对比两种材料的循环伏安图: (1)首周循环中, 红磷/导电碳黑复合材料在还原过程中的峰电流相 对更大, 意味着首次循环的不可逆容量更大; (2)在 三次循环扫描过程中，相对于基线电流，红磷/活性 炭复合材料均展示出更高的峰电流, 说明该材料具 有更高的活性物质利用率。因此可以预计红磷/活性 炭材料具有更大的实际容量和较小的首次不可逆容 量。在接近锂沉积电势处 ( $0.2 \mathrm{~V}$ 以下), 两种复合材 料都出现了小的还原峰, 再结合图 2(f)中红磷/活性 炭较差的循环寿命, 电化学性能测试中, 红磷/活性 炭复合材料的充放电及循环寿命的测试电势范围设 置为 $0.25 \sim 2.0 \mathrm{~V}$, 以避免锂金属的沉积。

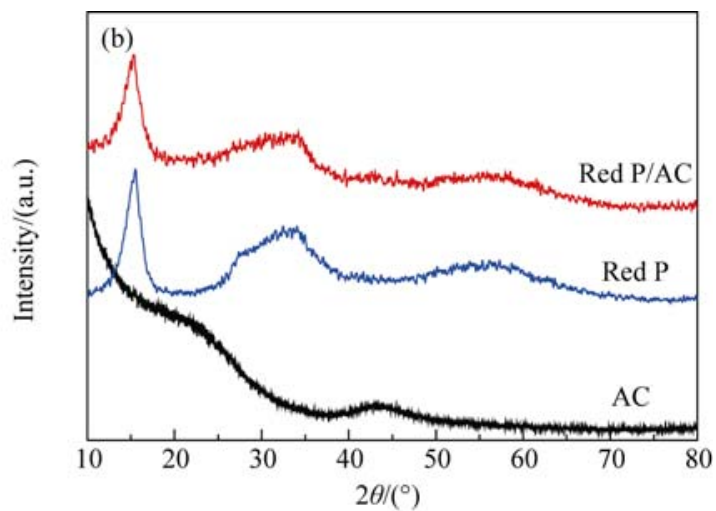

图 4 磷、碳和复合材料的 XRD 图谱

Fig. 4 XRD patterns of pure phosphorous, carbon and composites 

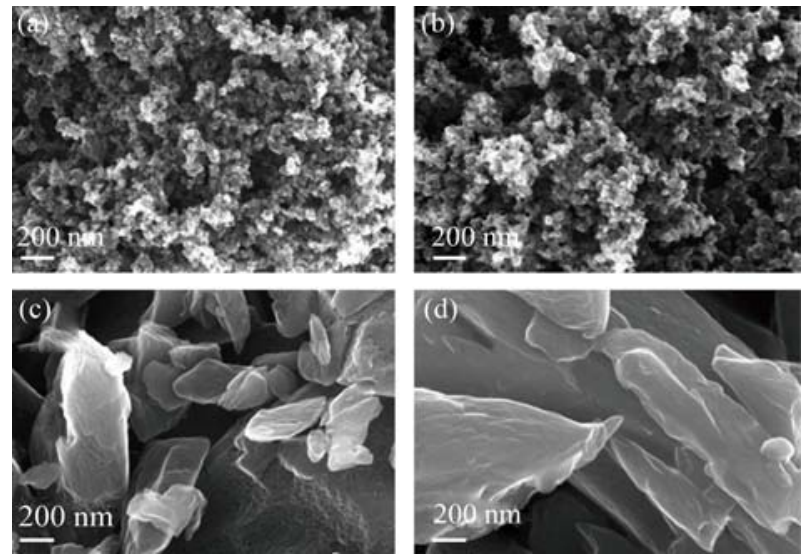

图 5 导电碳黑(a)、红磷/导电碳黑(b)、活性炭(c)和红磷/活性 炭(d)的 SEM 照片

Fig. 5 SEM images of BP2000 (a), P/BP2000 (b), AC (c) and P/AC (d)

图 7(a)和(b)是红磷/导电碳黑复合材料的前三 次充放电曲线和循环性能曲线, 图 7(c)和(d)为红磷/ 活性炭复合材料的前三次充放电曲线和循环性能曲
线。两种复合材料的充放电平台都在 $1.0 \mathrm{~V}$ 和 $0.75 \mathrm{~V}$ 左右 ${ }^{[25]}$ 。由于导电碳黑和活性炭等非活性材料在第 二次循环后都可以贡献 $200 \mathrm{mAh} / \mathrm{g}$ 的循环容量 (见 支持材料图 S1), 两种复合材料第二次循环后的循 环容量分别为 600 和 $1200 \mathrm{mAh} / \mathrm{g}$ 。因此扣除掉非活 性材料的容量贡献，两种复合材料的循环容量贡献 分别为 400 和 $1000 \mathrm{mAh} / \mathrm{g}$ 。基于含磷量 45.0\%可计 算红磷的嵌锂个数, 见图 7(a)和(b)上横轴。两种复 合材料的循环脱嵌锂个数分别为 1.0 和 2.8 。红磷/ 活性炭复合材料的实际脱嵌锂个数接近理论值 3.0 , 因此以气相沉积法制备的红磷/活性炭材料具有优 异的循环容量, 几乎所有的红磷都参与了 3 电子的 反应。而前者则只有 $1 / 3$ 红磷具有电化学活性。同 时, 相对于红磷/导电碳黑首次库伦效率 38.9\%, 红 磷/活性炭复合材料具有较高的首次库伦效率 82.5\%。随后循环的库伦效率超过了 $97.5 \%$ 。由图 7(b)和(d)的循环寿命图可以看出, 50 次循环后红磷/ 活性炭容量保持率为 $75.0 \%$, 红磷/导电碳黑复合材

表 1 碳基底和相应红磷/碳复合材料的比表面积和孔结构参数

Table 1 Surface area and pore-structure parameters of carbon substrates and corresponding red P/carbon composites

\begin{tabular}{|c|c|c|c|c|c|c|c|}
\hline \multirow{2}{*}{ Sample } & \multicolumn{3}{|c|}{$S /\left(\mathrm{m}^{2} \cdot \mathrm{g}^{-1}\right)$} & \multicolumn{2}{|c|}{$V /\left(\mathrm{cm}^{3} \cdot \mathrm{g}^{-1}\right)$} & \multicolumn{2}{|c|}{ Pore size/nm } \\
\hline & $S_{\mathrm{BET}}$ & $S_{\text {meso }}$ & $S_{\text {micro }}$ & $V_{\text {meso }}$ & $V_{\text {micro }}$ & $D_{\text {meso }}$ & $D_{\text {micro }}$ \\
\hline Blank BP2000 & 1128.1 & 758.2 & 369.9 & 0.86 & 0.57 & 2.00 & 0.63 \\
\hline Red P/BP2000 & 275.2 & 378.2 & 164.4 & 0.50 & 0.08 & 2.48 & 1.06 \\
\hline Subtracted value & 852.9 & 380 & 205.5 & 0.36 & 0.49 & - & - \\
\hline Blank AC & 1528.2 & 627.5 & 900.7 & 0.46 & 0.88 & 1.93 & 0.64 \\
\hline Red P/AC & 127.1 & 185.9 & 55.6 & 0.20 & 0.02 & 2.55 & 1.05 \\
\hline Subtracted value & 1401.1 & 441.6 & 844.4 & 0.26 & 0.86 & - & - \\
\hline
\end{tabular}
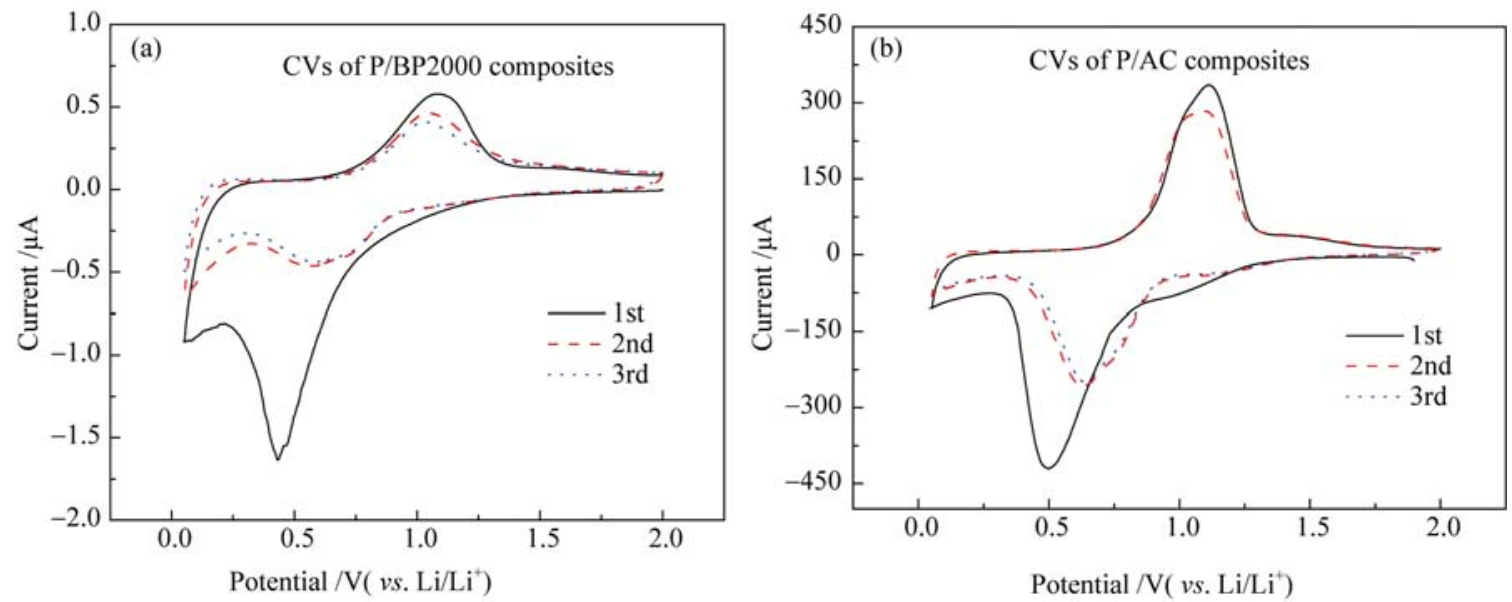

图 6 红磷/导电碳黑(a)和红磷/活性炭(b)复合材料的循环伏安曲线

Fig. 6 CV curves of P/BP2000 (a) and P/AC (b) composites 

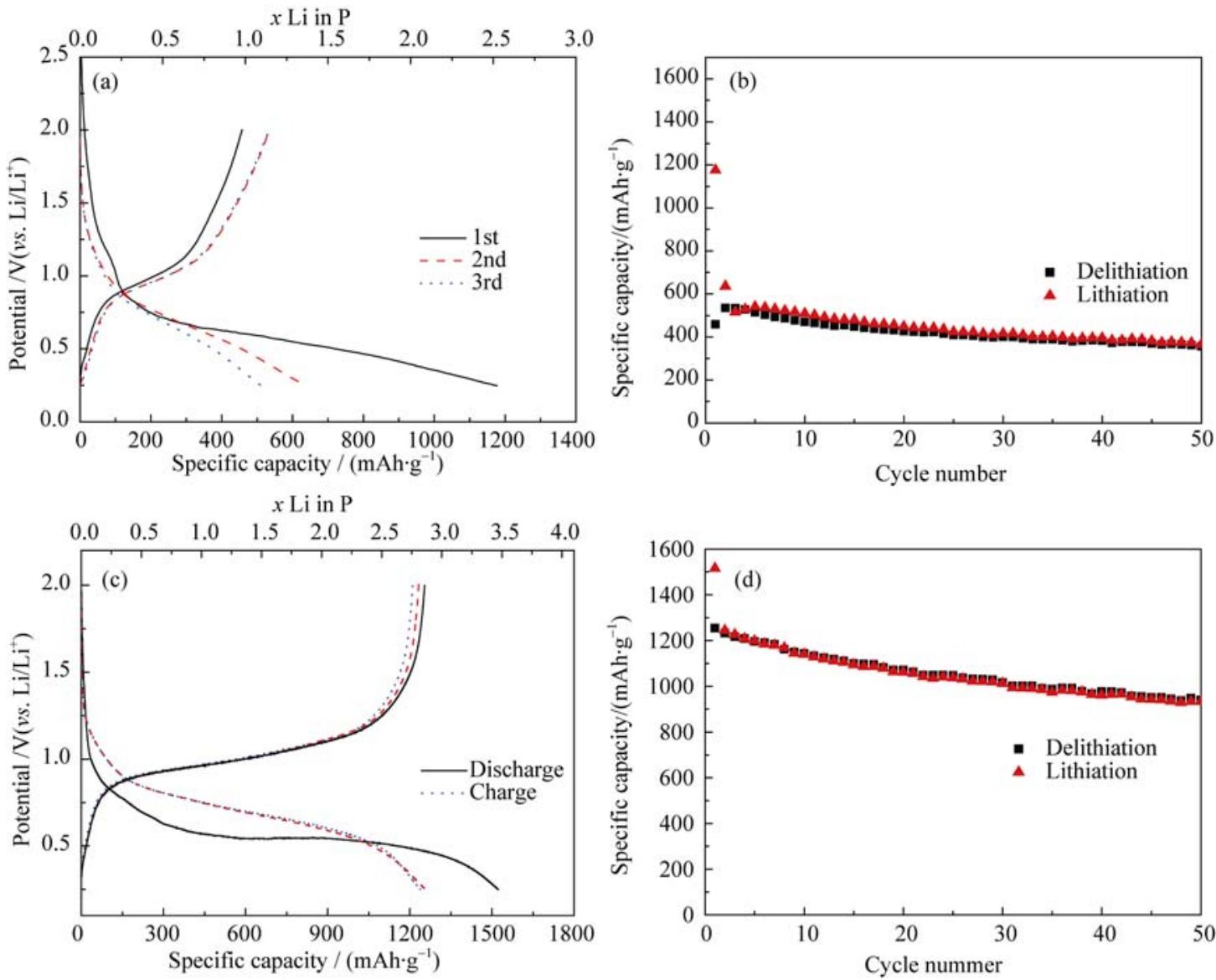

图 7 含磷量为 45\%红磷/导电碳黑复合材料的前三次充放电曲线(a)和循环性能曲线(b); 45\%红磷/活性炭复合材料的前三次充放 电曲线图(c) 和循环性能曲线(d)

Fig. 7 Initial three charge-discharge curves (a) and cycle performance (b) of P/BP2000 composite (45\%), initial three charge-discharge curves (c) and cycle performance (d) of P/AC composite (45\%)

料为 66.7\%。上述结果表明气相沉积法制备的红磷/ 碳复合材料展示了优异的比容量, 高首次库伦效率 以及稳定的循环性能, 其电化学性能均优于行星球 磨制备的红磷/活性炭复合材料。综合上述分析结果 进一步证明: 气相沉积法有效利用了碳基底的微孔 结构将红磷颗粒尺寸限制在纳米尺度，从而提高了 红磷的有效利用率。而行星式球磨法制备的复合材 料中红磷颗粒为微米尺寸。

结合两种复合材料的形貌和负载磷前后 BET 测试数据的变化可知, 尽管导电碳黑具有更小的粒 径和相似的孔容, 但两种碳中介孔和微孔的占比不 同, 造成红磷颗粒的尺寸分布不同。这是导致这两 种复合材料电化学性能不同的主要原因。小于 $2 \mathrm{~nm}$ 的微孔具有更强的吸附力, 可以把红磷的反应限制 在孔内。同时, 红磷锂化和去锂化过程的体积膨胀 而引起活性物质与碳基底之间松脱的问题可以得到 较大的抑制。介孔通常有较好的导液功能, 其相对 较大的空间和较小的吸附力对体积涨缩导致的磷碳
间附着情况有较大影响。这与两种复合材料在首次 循环中的表现一致。在首次红磷锂化膨胀过程中两 者都具有较高的利用率, 但在首次脱锂体积收缩过 程中表现迥异。以微孔负载为主的红磷/活性炭复合 材料表现了良好的电化学性能, $82.5 \%$ 的首次库伦 效率, 2.8 个电子的循环容量以及 50 次循环 $75.0 \%$ 的 容量保持率。

\section{3 结论}

通过球磨法和气相沉积法制备了锂离子电池负 极用磷/碳复合材料, 研究了电解液组成对电极材料 性能的影响, 优选出锂盐为 $1 \mathrm{~mol} / \mathrm{L} \mathrm{LiPF}$, 溶剂为 EC:EMC:DMC (1:1:1 V/V) 的混合酯类电解液。采用 行星式球磨法制备了 45\%含磷量的红磷/活性炭复合 材料。气相沉积法制备的含磷量均为 $45.0 \%$ 的红磷/ 导电碳黑和红磷/活性炭复合材料。其中, 气相沉积 法制备的两种磷/碳复合材料均表现了可逆的锂化和 
去锂化行为。红磷/活性炭复合材料表现了更好的首 次库伦效率(82.5\%), 接近理论容量的实际循环容量 (1200 mAh/g) 以及较好的循环稳定性。以气相沉积法 制备的红磷/碳复合材料, 利用了碳丰富的孔结构控 制红磷的尺寸分布, 有效解决了红磷因不导电而造 成的活性物质利用率低的问题，是制备高效红磷复 合材料的有效途径之一。

\section{参考文献:}

[1] LEE S W, YABUUCHI N, GALLANT B M, et al. High-power lithium batteries from functionalized carbon-nanotube electrodes. Nature Nanotechnology, 2010, 5(7): 531-537.

[2] JI L W, LIN Z, ALCOUTLABI M, et al. Recent developments in nanostructured anode materials for rechargeable lithium-ion batteries. Energy \& Environmental Science, 2011, 4(8): 2682-2699.

[3] WANG Y L, SUN Q L, ZHAO Q Q, et al. Rechargeable lithium/ iodine battery with superior high-rate capability by using iodinecarbon composite as cathode. Energy \& Environmental Science, 2011, 4(10): 3947.

[4] HOU P Y, ZHANG L Q, GAO X P. A high-energy, full concentrationgradient cathode material with excellent cycle and thermal stability for lithium ion batteries. Journal of Materials Chemistry A, 2014, 2(40): 17130-17138.

[5] GAO X P, YANG H X. Multi-electron reaction materials for high energy density batteries. Energy \& Environmental Science, 2010, 3(2): 174.

[6] KANG K. Electrodes with high power and high capacity for rechargeable lithium batteries. Science, 2006, 311(5763): 977-980.

[7] LI X, LIU J, BANIS M N, et al. Atomic layer deposition of solid-state electrolyte coated cathode materials with superior high-voltage cycling behavior for lithium ion battery application. Energy \& Environmental Science, 2014, 7(2): 768.

[8] WANG W G, WANG X, TIAN L Y, et al. In situ sulfur deposition route to obtain sulfur-carbon composite cathodes for lithium-sulfur batteries. Journal of Materials Chemistry A, 2014, 2(12): 4316.

[9] WANG W, LI G C, WANG Q, et al. Sulfur-polypyrrole/graphene multi-composites as cathode for lithium-sulfur battery. Journal of the Electrochemical Society, 2013, 160(6): A805-A810.

[10] BHASKAR A, DEEPA M, RAO T N. Size-controlled $\mathrm{SnO}_{2}$ hollow spheres via a template free approach as anodes for lithium ion batteries. Nanoscale, 2014, 6(18): 10762-10771.

[11] YU ZHEN-JUN,WANG YAN-LI,DENG HONG-GUI, et al. Synthesis and electrochemical performance of $\mathrm{SnO}_{2} /$ Graphene anode material for lithium ion batteries. Journal of Inorganic Materials, 2013, 28(5): 515-520.

[12] NOH J H, LEE K Y, LEE J K. Electrochemical characteristics of phosphorus doped Si-C composite for anode active material of lithium secondary batteries. Transactions of Nonferrous Metals Society of China, 2009, 19(4): 1018-1022.

[13] ZHU X, NING G, MA X, et al. High density $\mathrm{Co}_{3} \mathrm{O}_{4}$ nanoparticles confined in a porous graphene nanomesh network driven by an electrochemical process: ultra-high capacity and rate performance for lithium ion batteries. Journal of Materials Chemistry A, 2013, 1(44): 14023.

[14] PARK H C, LEE K H, LEE Y W, et al. Mesoporous molybdenum nitride nanobelts as an anode with improved electrochemical properties in lithium ion batteries. Journal of Power Sources, 2014, 269: $534-541$.

[15] STAN M C, KLÖPSCH R, BHASKAR A, et al. $\mathrm{Cu}_{3} \mathrm{P}$ binary phosphide: synthesis via a wet mechanochemical method and electrochemical behavior as negative electrode material for lithium-ion batteries. Advanced Energy Materials, 2013, 3(2): 231-238.

[16] CARENCO S, SURCIN C, MORCRETTE M, et al. Improving the Li-electrochemical properties of monodisperse $\mathrm{Ni}_{2} \mathrm{P}$ nanoparticles by self-generated carbon coating. Chemistry of Materials, 2012, 24(4): 688-697.

[17] BOYANOV S, ANNOU K, VILLEVIEILLE C, et al. Nanostructured transition metal phosphide as negative electrode for lithiumion batteries. Ionics, 2007, 14(3): 183-190.

[18] CHEN X, LI X, DING F, et al. Conductive rigid skeleton supported silicon as high-performance Li-ion battery anodes. Nano Letters, 2012, 12(8): 4124-4130.

[19] YUE L, ZHANG L, ZHONG H. Carboxymethyl chitosan: a new water soluble binder for Si anode of Li-ion batteries. Journal of Power Sources, 2014, 247: 327-331.

[20] GUO C, WANG D, LIU T, et al. A three dimensional $\mathrm{SiO}_{x} / \mathrm{C} @ \mathrm{RGO}$ nanocomposite as a high energy anode material for lithium-ion batteries. Journal of Materials Chemistry A, 2014, 2(10): 3521.

[21] WEN Z S, YANG J, WANG B F, et al. High capacity silicon/ carbon composite anode materials for lithium ion batteries. Electrochemistry Communications, 2003, 5(2): 165-168.

[22] PENG PENG, LIU YU, WEN ZHAO-YIN. Electrochemical performance of silicon/carbon/graphite composite anode for lithium ion batteries. Journal of Inorganic Materials, 2013, 28(11): 1195-1199.

[23] DU F H, WANG K X, FU W, et al. A graphene-wrapped silverporous silicon composite with enhanced electrochemical performance for lithium-ion batteries. Journal of Materials Chemistry A, 2013, 1(43): 13648.

[24] WANG L, HE X, LI J, et al. Nano-structured phosphorus composite as high-capacity anode materials for lithium batteries. Angewandte Chemie International Edition, 2012, 51(36): 9034-9037.

[25] QIAN J, QIAO D, AI X, et al. Reversible 3-Li storage reactions 
of amorphous phosphorus as high capacity and cycling-stable anodes for Li-ion batteries. Chemical Communications, 2012, 48(71): 8931.

[26] MARINO C, DEBENEDETTI A, FRAISSE B, et al. Activatedphosphorus as new electrode material for Li-ion batteries. Electrochemistry Communications, 2011, 13(4): 346-349.

[27] MARINO C, BOULET L, GAVEAU P, et al. Nanoconfined phosphorus in mesoporous carbon as an electrode for Li-ion batteries: performance and mechanism. Journal of Materials Chemistry, 2012, 22(42): 22713.

[28] KIM S, FANG S, ZHANG Z, et al. The electrochemical and local structural analysis of the mesoporous $\mathrm{Li}_{4} \mathrm{Ti}_{5} \mathrm{O}_{12}$ anode. Journal of Power Sources, 2014, 268: 294-300.

[29] ZHANG Z, ZHOU Z, NIE S, et al. Flower-like hydrogenated $\mathrm{TiO}_{2}(\mathrm{~B})$ nanostructures as anode materials for high-performance lithium ion batteries. Journal of Power Sources, 2014, 267: 388-393.

[30] TAO T, HE L, LI J, et al. Large scale synthesis of $\mathrm{TiO}_{2}$-carbon nanocomposites using cheap raw materials as anode for lithium ion batteries. Journal of Alloys and Compounds, 2014, 615: 1052-1055.

[31] GAO JIAN, MU XIN, LI JIAN-JUN, et al. Preparation and characterization of porous spherical $\mathrm{Li}_{4} \mathrm{Ti}_{5} \mathrm{O}_{12} / \mathrm{C}$ anode material for lithium ion batteries. Journal of Inorganic Materials, 2012, 27(3): 253-257.

[32] KIM J S, CHOI W, BYUN D, et al. Electrochemical characteristics of phosphorus doped silicon for the anode material of lithium secondary batteries. Solid State Ionics, 2012, 212: 43-46.

[33] SUN L Q, LI M J, SUN K, et al. Electrochemical activity of black phosphorus as an anode material for lithium-ion batteries. The Journal of Physical Chemistry C, 2012, 116(28): 14772-14779.

[34] KIM Y, PARK Y, CHOI A, et al. An amorphous red phosphorus/carbon composite as a promising anode material for sodium Ion batteries. Advanced Materials, 2013, 25(22): 3045-3049.

[35] QIAN J, WU X, CAO Y, et al. High capacity and rate capability of amorphous phosphorus for sodium ion batteries. Angewandte Chemie-International Edition, 2013, 52(17): 4633-4636.

[36] SUN S, GHIMBEU C M, JANOT R, et al. One-pot synthesis of $\mathrm{LiFePO}_{4}$-carbon mesoporous composites for Li-ion batteries. $\mathrm{Mi}$ croporous and Mesoporous Materials, 2014, 198: 175-184.

[37] XU H, DENG Y, SHI Z, et al. Graphene-encapsulated sulfur (GES) composites with a core-shell structure as superior cathode materials for lithium-sulfur batteries. Journal of Materials Chemistry A, 2013, 1(47): 15142.

[38] WENG W, LIN H, CHEN X, et al. Flexible and stable lithium ion batteries based on three-dimensional aligned carbon nanotube/silicon hybrid electrodes. Journal of Materials Chemistry A, 2014, 2(24): 9306.

[39] BRUECKNER J, THIEME S, GROSSMANN H T, et al. Lithiumsulfur batteries: Influence of C-rate, amount of electrolyte and sulfur loading on cycle performance. Journal of Power Sources, 2014, 268: $82-87$. 


\section{附录}
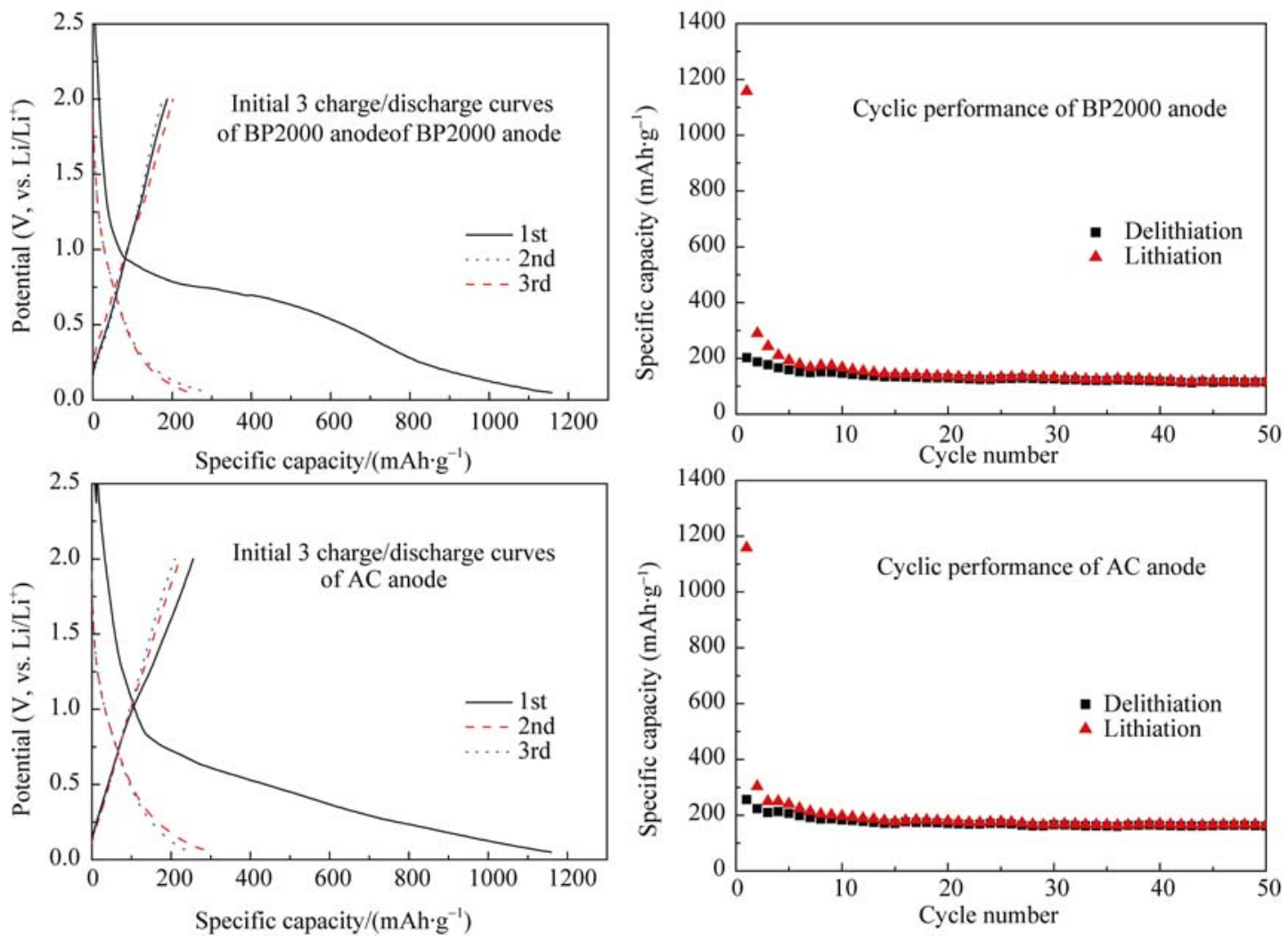

图 S1 非活性组分的容量贡献(a 和 b)导电碳黑空白极片(导电碳黑、PTFE 和乙炔黑, 质量比 38.5:10:20)的初始三周比容量和循 环寿命; (c 和 d)活性炭空白极片(活性炭、PTFE 和乙炔黑, 质量比 38.5:10:20)的初始三周比容量贡献和循环寿命

Fig. S1 The capacity contribution of inactive component. (a and b) The initial 3 charge/discharge curves and cycle performance of BP2000 blank anode (BP2000, PTFE and AB with weight ratio of 38.5:10:20). (c and d) The initial 3 charge/discharge curves and cycle performance of AC blank anode (AC, PTFE and AB with weight ratio of 38.5:10:20)

为了能较为准确的扣除红磷/碳复合材料中非活性组分 (包括碳基底、PTFE 和乙炔黑) 在复合材料循 环过程中提供的容量贡献, 对上述非活性组分做了相对应的电化学测试, 如图 S1。所测试的比容量是基于 非活性组分的质量计算。其中碳基底、PTFE 和乙炔黑的质量比等同于复合材料中相应组分的质量比 (28:10:20)。因而首次放电比容量为 $1200 \mathrm{mAh} / \mathrm{g}$, 可换算为基于红磷/碳复合材料的质量, 约为 $1174 \mathrm{mAh} / \mathrm{g}$ 。 首次充电之后迅速下降为近 $300 \mathrm{mAh} / \mathrm{g}$ 的容量贡献。在 5 次循环后, 比容量稳定在约 $200 \mathrm{mAh} / \mathrm{g}$ 。但对于 真实的磷/碳复合材料, 由于红磷负载后, 碳基底的比表面积大幅下降将导致非活性组分的容量贡献会较大 程度的下降。因而这些非活性组分在循环稳定后提供的容量将远低于空白极片所测试的 $200 \mathrm{mAh} / \mathrm{g}$ 。由图 S1 可以观察碳空白极片的首次不可逆容量达到 $900 \mathrm{mAh} / \mathrm{g}$ 。因而可以确定红磷/碳复合材料中较大的首次不 可逆容量应该是由其中的非活性组分提供的。

制备的红磷/碳复合材料的热重分析 (TGA) 如图 S2 所示。使用 TG (Mettler Toledo) 氩气流速 $50 \mathrm{~mL} / \mathrm{min}$ 下, 以 $10^{\circ} \mathrm{C} / \mathrm{min}$ 的升温速率测试其失重曲线。样品在 $420^{\circ} \mathrm{C}$ 有急剧失重, 此温度与红磷的升华温度相一致。 而其他温度范围未见明显重量损失。由此可以计算得红磷/导电碳黑和红磷/活性炭复合材料中红磷含量均为 45.0\%。图 3S 显示了红磷、碳和复合材料的拉曼光谱。测试光源为 $\mathrm{He}-\mathrm{Ne}$ 激光, 波长为 $514.5 \mathrm{~nm}$, 功率为 $25 \mathrm{~mW}$ 。由图中可以看出, 在红磷负载前后碳基底的 D 峰和 $\mathrm{G}$ 峰的波长都没有发生位移, 表明该复合材料 中红磷和碳没有强的相互作用 ${ }^{[26]}$ 。 

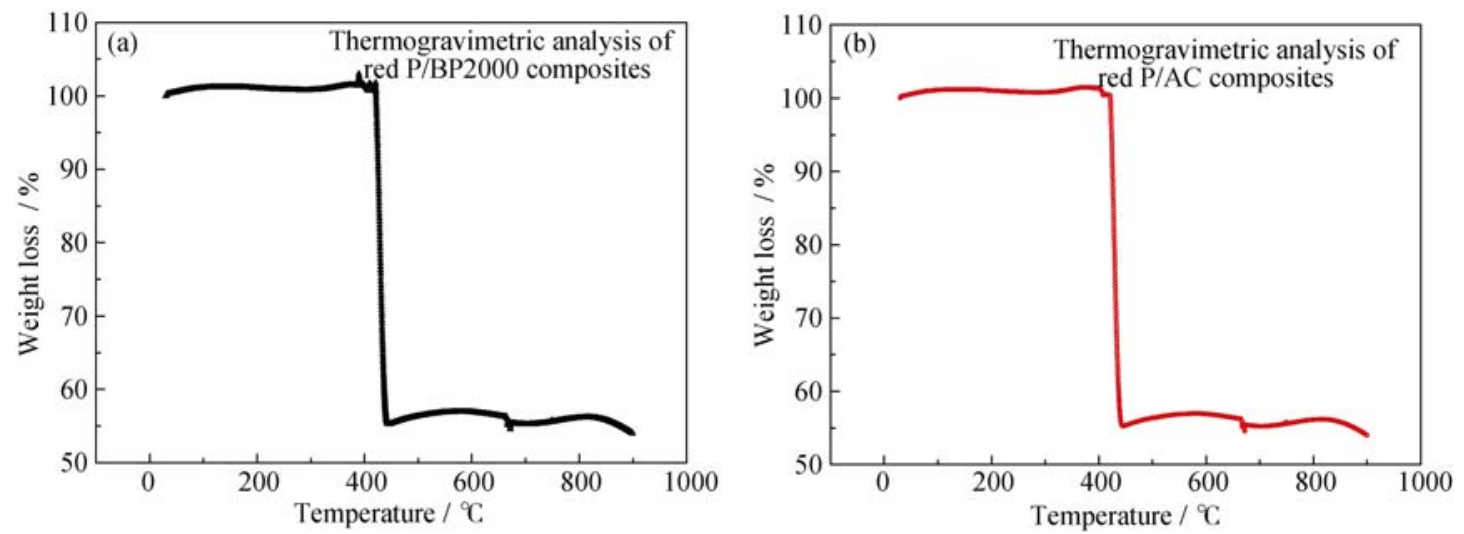

图 S2 红磷/导电碳黑(a)和红磷/活性炭(b)复合材料的热重曲线

Fig. S2 TGA of the as-prepared red P/BP2000(a)and red P/AC(b)composites

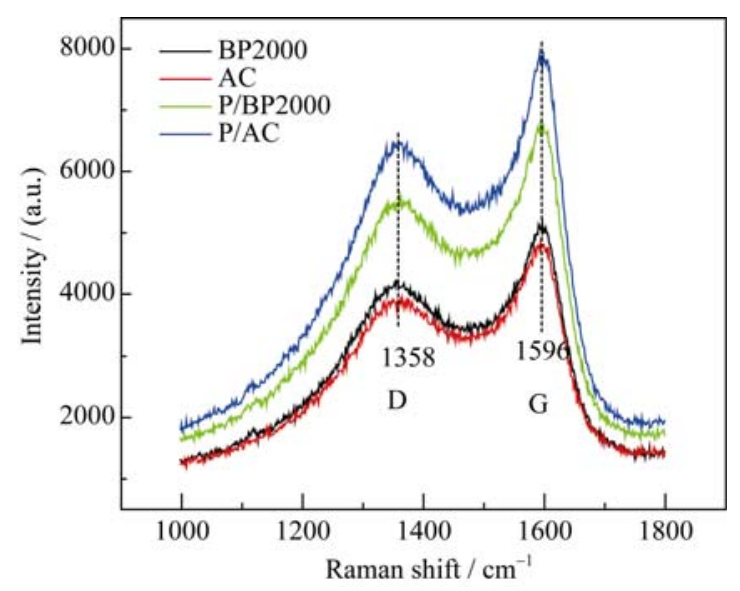

图 S 3 碳和复合材料的拉曼光谱

Fig. S 3 Raman Spectra of pure carbon and composites 